\title{
Ventricular arrhythmia and sudden cardiac death in young Leonbergers
}

\section{Wiberg, M.}

2020-02

Wiberg , M , Niskanen , J E , Hytönen , M , Dillard , K, Hagner , K , Anttila , M \& Lohi , H 2020 , ' Ventricular arrhythmia and sudden cardiac death in young Leonbergers ' , Journal of Veterinary Cardiology , vol. 27 , pp. 10-22 . https://doi.org/10.1016/j.jvc.2019.11.006

http://hdl.handle.net/10138/322099

https://doi.org/10.1016/j.jvc.2019.11.006

cc_by_nc_nd

acceptedVersion

Downloaded from Helda, University of Helsinki institutional repository.

This is an electronic reprint of the original article.

This reprint may differ from the original in pagination and typographic detail.

Please cite the original version. 


\title{
Ventricular arrhythmia and sudden ${ }^{a}$ cardiac death in young Leonbergers
}

\author{
Q5 M. Wiberg, DVM, PhD ${ }^{a, *}$, J.E. Niskanen, $M S^{b}{ }^{b}$, M. Hytönen, \\ $\mathrm{PhD}^{\mathrm{b}}$, K. Dillard, DVM ${ }^{\mathrm{b}, \mathrm{d}}$, K. Hagner, DVM ${ }^{\mathrm{c}}$, M. Anttila, DVM, \\ $\mathrm{PhD}^{\mathrm{d}}, \mathrm{H}$. Lohi, $\mathrm{PhD}^{\mathrm{b}}$
}

${ }^{a}$ Department of Equine and Small Animal Medicine, Faculty of Veterinary Medicine, University of Helsinki, P.O BOX 57, 00014, Helsinki, Finland

${ }^{\mathrm{b}}$ Department of Veterinary Biosciences, Department of Medical Genetics, Medicum, University of Helsinki, Folkhälsan Research Center, Helsinki, Haartmaninkatu 8, 00290, Helsinki, Finland

${ }^{\mathrm{C}}$ Veterinary Pathology and Parasitology, Department of Veterinary Biosciences, Faculty of Veterinary Medicine, University of Helsinki, P.O. Box 66, 00014, Helsinki, Finland ${ }^{\mathrm{d}}$ Veterinary Bacteriology and Pathology Research Unit, Finnish Food Authority, P.O.

Q1 BOX 200, 00027, Helsinki, Finland

Received 31 May 2019; received in revised form 18 November 2019; accepted 18 November 2019
KEYWORDS
Inheritance;
Necropsy;
Cardiology;
Arrhythmia

\begin{abstract}
Objectives: To describe unexpected sudden cardiac death $(\mathrm{SCD})$ in young Leonbergers ( $<3$ years) and to review the circumstances before death and necropsy findings; to prospectively evaluate the presence of possible arrhythmias in young Leonbergers; and to examine pedigrees for determining potential modes of inheritance.

Animals: Postmortem evaluations included 21 Leonbergers. Clinical evaluation consisted of 46 apparently healthy Leonbergers with and without a close family history of SCD.

Materials and methods: Necropsy reports were reviewed retrospectively. Prospective clinical evaluation included physical examination, 5-min electrocardiogram, 24-h Holter, echocardiography, and laboratory tests. Pedigree data were examined for mode of inheritance.

Results: Based on necropsy reports, SCD occurred at a median age of 12 months (range, 2.0-32.0 months) without any previous clinical signs and usually in rest. No evidence of structural cardiac disease was found; arrhythmia-related death was suspected. Clinical evaluation and 24-h Holter showed ventricular arrhythmia
\end{abstract}

\footnotetext{
* Corresponding author.

E-mail address: maria.wiberg@helsinki.fi (M. Wiberg).
} 
M. Wiberg et al.

(VA) in 14 apparently healthy Leonbergers (median age, 18 months; range, 12-42 months). Severity of VA varied from infrequent couplets/triplets to frequent complexity (couplets, triplets, non-sustained ventricular tachycardias, (VTs) characterized by polymorphology. During follow-up, two dogs with polymorphic VT died. Although breed specificity and high prevalence indicate a heritable disease, based on available pedigree data, the mode of inheritance could not be determined.

Conclusions: Sudden cardiac death in young Leonbergers is associated with malignant VA characterized by complexity and polymorphic nature. Diagnosis is based on 24-h Holter monitoring. Pedigree analysis suggests that the arrhythmia is familial.

(c) 2019 Elsevier B.V. All rights reserved.

\begin{tabular}{ll}
\multicolumn{2}{l}{ Abbreviations } \\
BW & body weight \\
EPSS & E-point-to-septal separation \\
HR & Heart rate \\
IVSd & $\begin{array}{l}\text { Interventricular septum thickness at } \\
\text { end-diastole }\end{array}$ \\
& $\begin{array}{l}\text { Interventricular septum thickness at } \\
\text { IVSs }\end{array}$ \\
& end-systole \\
LVEDV & Left ventricular end-diastolic volume \\
LVESV & Left ventricular end-systolic volume \\
LVIDd & Left ventricular internal dimension \\
& at end-diastole \\
RPS & right parasternal \\
SA & short axis \\
SCD & Sudden cardiac death \\
VA & Ventricular arrhythmia \\
VPC & Ventricular premature complex \\
VT & Ventricular tachycardia $(\geq 4$ VPCs in \\
& a row)
\end{tabular}

\section{Introduction}

Sudden death is defined as an unexpected fatal event occurring without any previous clinical signs in apparently healthy individuals [1-3]. Cardiovascular disorders are the most common reason for sudden death in humans [2]. Sudden cardiac death (SCD) may be associated either with structural cardiac disease or with primary electric disorders $[1,2,4]$.

In dogs, SCD has been reported with congenital cardiac anomalies, certain hereditary cardiomyopathies, and acquired myocardial disease [5-8]. Dilated cardiomyopathy (DCM) and arrhythmogenic cardiomyopathy are familial diseases typically with adult onset of clinical signs $[7,9,10]$. The presence of ventricular arrhythmias (VAs) may lead to sudden death with or without preceding signs $[6,7,11,12]$. An uncommon juvenile form of DCM reported in the Portuguese Water Dog may cause sudden death at less than one year of age [13].

Arrhythmia-related sudden death in the absence of structural cardiac disease is rarely reported in dogs $[14,15]$. Inherited ventricular arrhythmia (IVA) has been described in young German Shepherd dogs (GSDs) and is characterized by polymorphic rapid ventricular tachycardia (VT) and sudden death [14]. A recent study reported VA in a family of Rhodesian Ridgebacks [15]. In young humans, fatal primary arrhythmias are usually caused by familial ion channelopathies $[1,3]$. In dogs, a primary channelopathy called long QT syndrome has been reported in a family of English Springer Spaniels [16].

We have recently observed several Leonbergers that died unexpectedly at young age without any evidence of structural cardiac disease. To our knowledge, SCD in this breed has not been previously described. In this study, we retrospectively reviewed necropsy reports of Leonbergers $<3$ years old that died suddenly with macroscopically normal heart and no microscopic myocardial changes. A prospective clinical evaluation was also performed to identify possible arrhythmias in Leonbergers with and without a close family history of SCD. Finally, we sought to determine the mode of inheritance.

\section{Animals, materials, and methods}

\section{Anatomic pathology}

Necropsy reports of Leonbergers were reviewed from 2008 to 2018. The inclusion criterion was a sudden unexpected death at the age $<3$ years without any specific organ changes apart from acute pulmonary congestion and edema. The 
necropsies were conducted at the Veterinary Bacteriology and Pathology Research Unit, Production and Companion Animal Pathology Section, Finnish Food Safety Authority, Helsinki and at Veterinary Pathology and Parasitology, Department of Veterinary Biosciences, Faculty of Veterinary Medicine, University of Helsinki. All dogs were sent for necropsy by the owner. Age, gender, body weight $(\mathrm{BW})$, circumstances associated with death, possible preceding clinical signs (if reported), and macroscopic and histological findings were documented. Complete necropsy was performed on all dogs included in this study. Tissue samples were acquired from all major organs, fixed in neutral-buffered $10 \%$ formalin, routinely processed, sectioned at $4 \mu \mathrm{m}$ thickness, and stained with hematoxylin and eosin for histological examination by light microscopy. The heart weight to $B W$ ratio was calculated in cases in which the heart weight was included in the postmortem report.

\section{Clinical evaluation}

Leonbergers $<4$ years of age were recruited to participate in a prospective clinical study. The clinical study included dogs that were close relatives to SCD dogs and dogs without a known family history of SCD. All examinations were performed at the University Small Animal Hospital, University of Helsinki between 2015 and 2018. Informed owner consent was obtained. The clinical study included a detailed case history that was obtained by owner interview. All dogs underwent a physical examination, 5-min electrocardiogram (ECG), 24-h Holter recording, and conventional echocardiography. Blood samples were collected for complete blood cell count and serum biochemistry profile. Additional laboratory tests included serum high-sensitivity troponin I measurement $\left(\mathrm{hs}-\mathrm{Tnl}^{\mathrm{e}}\right)$ and Snap4Dx test. ${ }^{f}$

This study was approved by the Animal Ethics Committee of the State Provincial Office of Southern Finland (ESAVI/8176/04.10.07/2016).

\section{Five-minute electrocardiogram}

Dogs were either positioned on the floor in right or left lateral recumbency or standing during the $5 \mathrm{~min}$ of a six-channel electrocardiogram. The

\footnotetext{
${ }^{\mathrm{e}}$ ADVIA Centaur Tnl-Ultra, Siemens Healthcare Diagnostics Inc., Eschborn, Germany; run by IDEXX Laboratories, Ludwigsburg, Germany.

f IDEXX Laboratories, Ludwigsburg, Germany.
}

presence and number of premature beats (ventricular and supraventricular) were recorded.

\section{Holter}

A digital Holter recorder ${ }^{g}$ that uses a six-lead system was used to obtain 24-h electrocardiogram. Two electrode patches were placed in each hemithorax side and the device was covered with elastic bandages. All dogs wore the monitor for $24 \mathrm{~h}$ in their home environment and the owners were asked to mark the dogs activities in a diary. Televet software, version $6.0^{\mathrm{h}}$ was used for analysis. The data were analyzed with automatic analysis settings and manually by an experienced veterinarian (M.W.). The following parameters were identified and measured: hours recorded, minimum, maximum, and mean heart rate (HR) during the recording, number of ventricular premature complexes (VPCs), ventricular couplets, triplets and VT (defined as $\geq 4$ VPC in a row, nonsustained VT $<30 \mathrm{~s}$ duration), presence of sinus pauses (defined as RR interval $>3.0 \mathrm{~s}$ ), presence of supraventricular premature complexes, ventricular escape complexes (defined as a wide QRS complex occurring after a sinus pause), and second-degree atrioventricular block (defined as a single $\mathrm{P}$ wave not related to QRS complex).

Based on the severity of the arrhythmias detected in the initial Holter monitoring, the dogs were classified retrospectively in clinical study groups as follows: VA-affected group ( $>100$ VPCs, and/or multiple couplets, triplets, or $\geq 1$ non-sustained VT period), VA-borderline group (single VPCs $<100$ and one to two couplets/triplets but no VTs) and VA-unaffected group ( $<50$ VPCs and no couplets or triplets or VT).

To assess possible abnormalities for QT intervals, Holter recordings were used to determine complex durations from all 46 dogs. From each Holter recording, three consecutive RR, $\mathrm{PQ}$, and QT intervals were measured during the sinus rhythm in heart rates of $90-110$ beats per minute (bpm), using lead II and paper speed $50 \mathrm{~mm} / \mathrm{s}$. The mean RR, PQ, and QT intervals were calculated for each dog. The mean RR, PQ, and QT intervals were calculated for the clinical study groups.

\section{Echocardiography}

A complete standard transthoracic echocardiography was performed on all 46 dogs by a single

\footnotetext{
s Televet 100, Engel Engineering Services GmbH, Germany.

h Televet 100, software version 6.0; Engel Engineering Services $\mathrm{GmbH}$, Germany.
} 
M. Wiberg et al.

operator (M.W.) using an ultrasonographic unit with a $1-5 \mathrm{MHz}$ transducer. Examinations were performed without sedation in right and left lateral recumbency or in standing position with simultaneous lead II electrocardiogram recording. Standard echocardiographic views were obtained and measurements were made in accordance with the available guidelines [17-19]. Three representative consecutive cardiac cycles were measured and averaged.

The left ventricular end-diastolic volume (LVEDV) and left ventricular end-systolic volume (LVESV) were calculated in the right parasternal (RPS) long-axis four-chamber view using Simpson's modified method of discs. Left ventricular volume was indexed to body surface area [20]. Left ventricular dimensions; left ventricular internal dimension at end-diastole (LVIDd), left ventricular internal dimension at end-systole, interventricular septum thickness at end-diastole (IVSd), interventricular septum thickness at end-systole (IVSs), left ventricular posterior wall thickness at enddiastole, left ventricular posterior wall thickness at end-systole were measured using the $M$-mode from the RPS short-axis (SA) view from chordae tendineae level. E-point-to-septal separation (EPSS) was calculated from the RPS SA M-mode at the level of mitral valve. The left ventricular sphericity index was measured by dividing left ventricular length by the LVIDd obtained from the M-mode image. The left atrial diameter, aortic root diameter, and the ratio of the left atrial diameter to aortic root diameter were measured from the RPS SA view at the level of aortic valve in early diastole [21]. The aortic hinge diameter was measured in early systole from the RPS long-axis view at the level of the valve leaflet hinge points. An average of three systolic aortic and pulmonary flow velocities were measured with continuous and pulse wave Doppler. Aortic velocities were obtained from the subcostal view or from left apical view [22] and pulmonic velocities from the RPS SA view. Mitral and tricuspid valves were examined for regurgitation using color flow mapping. The severity of the mitral valve regurgitation (MR) was assessed using MR jet area/left atrium area \% [23].

\section{Pedigree analysis}

The pedigree data for SCD dogs and dogs included in the clinical study were obtained from the Finnish Kennel Club public database (http://jalostus. kennelliitto.fi). The pedigree was constructed

\footnotetext{
ie33 and EPIQ7, Philips Ultrasound, USA.
}

using the AncesTrim software [24] and rendered using GenoPro (version 2.5.4.1, http://www. genopro.com).

\section{Statistics}

Normality testing was performed by the ShapiroWilk test of normality and normal Q-Q plots for all continuous study variables. Mean \pm SD (normally distributed variables) and median + range (non-normally distributed variables) were presented for the total postmortem population and for the total clinical study population and for the clinical study groups (VA-affected group, VAborderline group, VA-unaffected group). Echocardiographic variables for the VA-unaffected group were also reported by gender.

The relationships between the echocardiographic variables and the background variables (age, gender, and BW) for the total clinical population and separately within the VA-unaffected group were analyzed with linear regression (age and BW) and with independent samples t-test (gender). Each background variable was analyzed separately. In case of more than one background variable was found significant in the univariate analysis, multivariate statistical models were constructed including all the significant background variables.

The clinical study groups (VA-affected group, VA-borderline, and VA-unaffected) were then compared with the echocardiographic variables with either analysis of variance models or analysis of covariance models depending on the results of the analyses regarding the background variables. Statistically significant background variables were included in these models together with the study group as fixed effects. If none of the background variables for a particular echocardiographic variable was statistically significant in the earlier analysis, the model had only the study group as the sole fixed effect.

All statistical analyses were performed using commercially available software. ${ }^{j} p$-values $<0.05$ were considered statistically significant.

\section{Results}

\section{Anatomic pathology}

Twenty-one Leonbergers (13 males and 8 females) with complete necropsy were included. The

\footnotetext{
${ }^{\mathrm{j}}$ SAS ${ }^{\circledR}$ System for Windows 9.4; SAS Institute Inc, Cary, North Carolina, USA.
} 
median age at death was 12 months (range, 2.0-32.0 months). Median BW for 15 of 21 dogs was $50.0 \mathrm{~kg}$ (range, 6.4-60.0 kg). Sudden death without any preceding clinical signs was reported in 20 of 21 dogs. The sudden collapse usually occurred when the dogs were at rest for a longer period or when they were resting after exercise. Two of these 21 dogs had taken part in the clinical evaluation study and had been diagnosed with severe VA but did not show any clinical signs before death that occurred 8-11 months later. Dog number 1 (Table 1) died suddenly at 32 months of age, whereas dog number 4 (Table 1) was euthanized at the age of 21 months after an acute collapse with signs of shock and arrhythmia after vigorous exercise.

Necropsy findings of the 20 SCD dogs were consistent with acute heart failure with marked acute congestion and edema of the lungs and congestion in other organs (mainly liver). Cause of death was thought to be arrhythmia. The heart weight to BW ratio was calculated for 11 of 20 dogs. The median ratio was normal $(0.73$; range, $0.6-0.8)$ [25]. The hearts were macroscopically normal; and there were no specific histopathological changes in the myocardium, epicardium, endocardium, connective tissue, or blood vessels apart from one dog, in which there was mild to moderate lipomatosis in the right ventricle and septum and multifocal vacuolization of the cardiomyocytes.

There were no other significant organ changes present that could explain the sudden death. In four dogs (including dog numbers 1 and 4 in Table 1 ), the abdominal aorta appeared to be narrower than normal (diameter ranging from 10 to $11.8 \mathrm{~mm}$ measured cranial to the kidney or at diaphragmatic outlet). In one of these four dogs, there was hemosiderosis of the aortic wall with occasional organized thrombi in the aortic vasa vasorum and in the meningeal vessels. In the remaining 17 dogs, there were no changes noted in the abdominal aorta. In the macroscopic examination of dog number 4 (Table 1), myocardial petechiae and ecchymoses, hemorrhagic content in the stomach and intestine, and severe congestion in the mucosa of the stomach and jejunum were present and considered as caused by shock.

\section{Clinical evaluation}

A total of 46 Leonbergers (19 males and 27 females) were included in the clinical study. All dogs were clinically healthy without any previous history of clinical signs related to cardiac disease. The median age was 19.0 months (range, 9.0-42 months). The median BW of all dogs was $53.3 \mathrm{~kg}$ (range, 40.9-72.0 kg); median BW for males was $61.0 \mathrm{~kg}$ (range, $53.0-72.0 \mathrm{~kg}$ ) and for females $47.2 \mathrm{~kg}$ (range, 40.9-63.0 kg). Physical examination and auscultation did not reveal any significant findings. Mean HR during auscultation was $91.6 \pm 9.59 \mathrm{bpm}$. Complete blood cell count and serum biochemistry profile did not reveal any clinically significant findings.

\section{Holter}

A one-time Holter recording was performed on 46 Leonbergers. The quality of the recordings was considered good to satisfactory in all dogs. The median duration of the 46 recordings was $24 \mathrm{~h}$ (range, 21.0-26.5 h). Median minimum HR was 31 bpm (range, 21.0-63.0 bpm), maximum HR was $278.0 \mathrm{bpm}$ (range, 229.0-300.0 bpm), and mean HR was 79.5 bpm (range, 61-117.8 bpm). Sinus pauses $(>3.0 \mathrm{~s})$ were detected in 16 of 46 dogs (median number of pauses 4.0/Holter; range, 1-412.0). Ventricular escape complexes were observed in 20 of 46 dogs (median 1.0/Holter; range, 1-2800). Second-degree atrioventricular blocks were observed in 5 of 46 dogs (median 4; range, 1-40).

Based on the findings from initial Holter monitoring, 14 dogs (median age, 18 months and range, 12-42 months) were considered affected with VA. Based on the severity of VA, the dogs were stratified into a VA-affected group (7 dogs; 3 males, 4 females) and a VA-borderline group (7 dogs; 3 males, 4 females). The other 32 dogs were considered unaffected by VA (13 males, 19 females). There were no significant differences regarding age or BW between the clinical study groups.

The results of Holter monitoring for the VAaffected group are shown in Table 1. The median number of single VPCs in the VA group was 673.0 (range, 1.0-1760), couplets 66.0 (range, 0-830.0), triplets 12.0 (range, 0-125.0), and nonsustained VT period 3.0 (range, 0-101.0). Single VPCs and ventricular complexes were of either right bundle branch block or left bundle branch block morphology. Ventricular tachycardia periods were usually fast (mean maximum speed $>360$ bpm) and consisted of 4-12 VPCs. Tachycardias were either monomorphic with right bundle branch block type ( $57 \%$ of VTs) or left bundle branch block type $(15 \%)$ or polymorphic $(28 \%)$. Characteristic polymorphic VT is shown in Fig. 1. Single supraventricular premature complexes were found in some recordings. Ventricular arrhythmia was not found to be associated with exercise or increased $\mathrm{HR}$. The VA was most commonly detected in the slower heart rates $(<80 \mathrm{bpm})$ and during more 
Table 1 Characterization of ventricular arrhythmias detected by 24-h Holter monitoring in 14 Leonbergers.

\begin{tabular}{|c|c|c|c|c|c|c|c|c|c|c|c|}
\hline $\begin{array}{l}\text { Dog } \\
\text { (number) }\end{array}$ & $\begin{array}{c}\text { Age } \\
\text { (months) }\end{array}$ & Gender & $\begin{array}{c}\text { Holter } \\
\text { VPC (number) }\end{array}$ & $\begin{array}{c}\text { Couplet } \\
\text { (number) }\end{array}$ & $\begin{array}{c}\text { Triplet } \\
\text { (number) }\end{array}$ & $\begin{array}{c}\mathrm{VT} \\
\text { (number) }\end{array}$ & $\begin{array}{l}\text { Holter repeat } \\
\text { after months }\end{array}$ & $\begin{array}{c}\text { VPC } \\
\text { (number) }\end{array}$ & $\begin{array}{c}\text { Couplet } \\
\text { (number) }\end{array}$ & $\begin{array}{c}\text { Triplet } \\
\text { (number) }\end{array}$ & $\begin{array}{c}\mathrm{VT} \\
\text { (number) }\end{array}$ \\
\hline \multicolumn{12}{|c|}{ VA-affected } \\
\hline 1 & 21 & Male & 600 & 27 & 12 & 8 & * & & & & \\
\hline 2 & 29 & Female & 1127 & 67 & 28 & 3 & 2 & 802 & 36 & 15 & 13 \\
\hline 3 & 12 & Male & 1760 & 830 & 125 & 101 & 3 & 2892 & 978 & 315 & 159 \\
\hline 4 & 13 & Male & 390 & 89 & 68 & 38 & ** & & & & \\
\hline 5 & 15 & Female & 933 & 66 & 0 & 0 & 2 & 2558 & 86 & 1 & 0 \\
\hline 6 & 18 & Female & 673 & 0 & 0 & 0 & & & & & \\
\hline 7 & 15 & Female & 1 & 0 & 2 & 1 & & & & & \\
\hline \multicolumn{12}{|c|}{ VA-borderline } \\
\hline 8 & 30 & Female & 8 & 1 & 1 & 0 & 6 & 0 & 0 & 0 & 0 \\
\hline 9 & 42 & Female & 2 & 1 & 0 & 0 & & & & & \\
\hline 10 & 16 & Male & 3 & 1 & 0 & 0 & 18 & 2 & 0 & 0 & 0 \\
\hline 11 & 18 & Male & 9 & 1 & 0 & 0 & 12 & 3 & 0 & 0 & 0 \\
\hline 12 & 12 & Female & 1 & 0 & 1 & 0 & 6 & 4 & 0 & 0 & 0 \\
\hline 13 & 30 & Female & 10 & 1 & 0 & 0 & & & & & \\
\hline 14 & 38 & Male & 96 & 2 & 0 & 0 & 6 & 23 & 0 & 0 & 0 \\
\hline
\end{tabular}

Based on the severity of the ventricular arrhythmia (VA), dogs were classified to a VA-affected group ( $>100$ single VPCs, and/or multiple couplets, triplets, or $>1$ non-sustained VT period) and to VA-borderline group (single VPCs $<100$ and one to two couplets/triplets but no VTs). The results of the first and repeat Holter are shown. *SCD after 11 months, **euthanasia after 8 months.

VT, ventricular tachycardia; VPCs Ventricular premature complexes. 


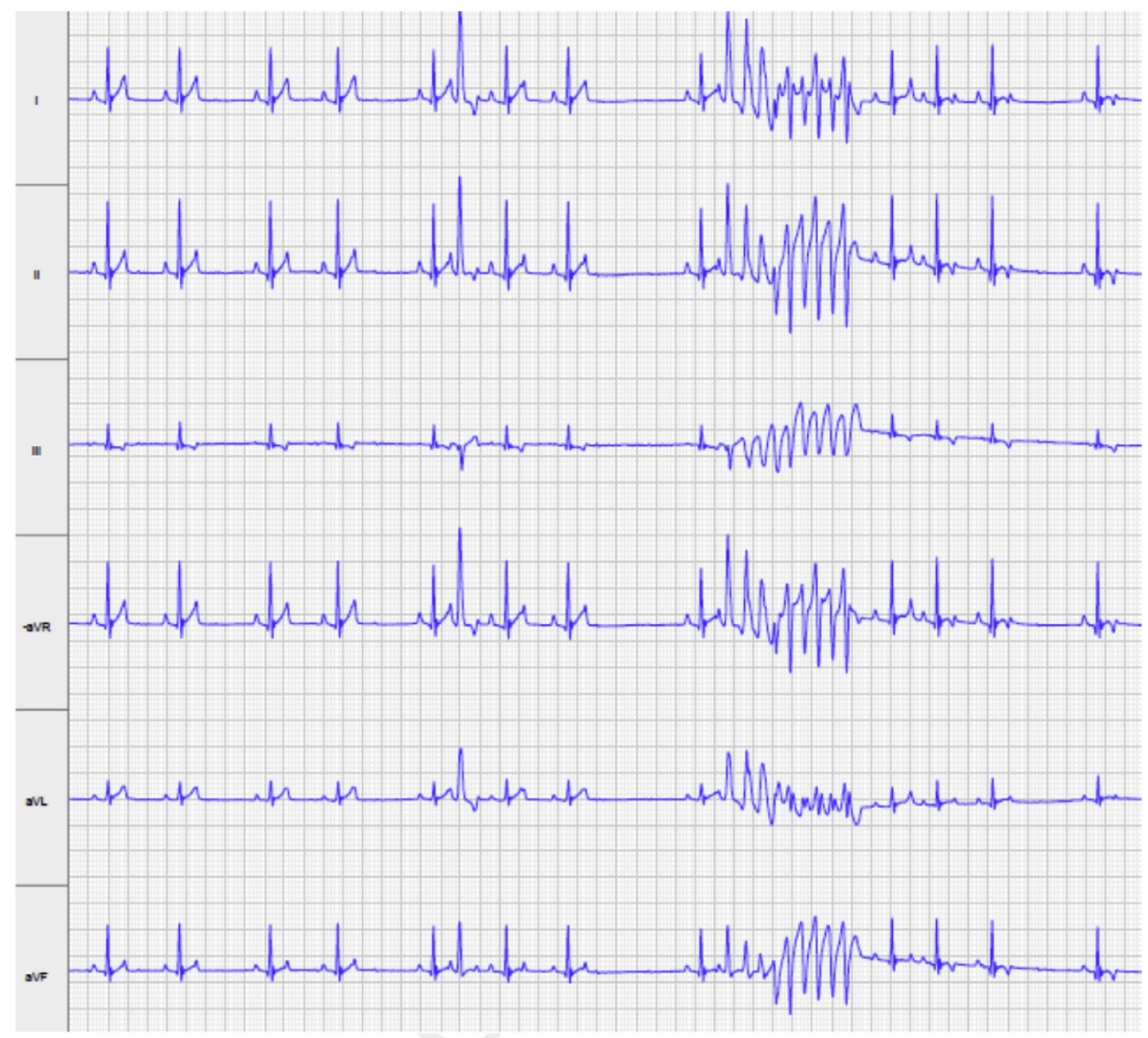

Fig. 1 Six-lead ECG (Holter monitoring) of a Leonberger diagnosed with ventricular arrhythmia. Dog number 4 (Table Q7 1).Paper speed: $25 \mathrm{~mm} / \mathrm{s}$. Amplitude: $5 \mathrm{~mm} / 1 \mathrm{mV}$. ECG, electrocardiogram.

profound sinus arrhythmia during rest and at rest after exercise. Although tachycardia periods were often associated with a preceding pause, VT without preceding pause was also detected. Single ventricular escape complexes were detected in some dogs; dog number 4 also displayed runs of accelerated idioventricular rhythm (rate $<130$ bpm).

All dogs in the VA group had blood samples taken for assays of serum hs-Tnl and Snap 4Dx. The mean hs-Tnl value was $0.099 \pm 0.157 \mathrm{mmol} / \mathrm{l}$; the values were in the normal laboratory reference range for 5 of 7 dogs $(<0.07 \mathrm{mmol} / \mathrm{l})$ and elevated in dog number $6(0.08 \mathrm{mmol} / \mathrm{l})$ and dog number 4 $(0.45 \mathrm{mmol} / \mathrm{l})$. A Snap 4DX test was positive for borreliosis in 1 of 7 dogs (dog number 2), but the repeat test was negative.

The dogs were not treated with any antiarrhythmic medications during the study. Follow-up Holter was performed on 3 of 7 VA-affected group dogs within 2-3 months. The results were similar to the dogs' initial Holter results with VA complexity (Table 1). Two of the affected dogs died 8 and 11 months after the clinical examination. Dog number 1 died during rest after exercise and dog number 4 was euthanized because of lifethreatening symptoms of severe shock and arrhythmia after a bout of vigorous exercise.

In the VA-borderline group, the median number of single VPCs in Holter of 7 dogs was 8.0 (range, $1-96)$; these dogs also had either one to two couplets (6 dogs) and or single triplet (2 dogs). Follow-up Holter was performed on 5 of 7 dogs in the borderline group in 6-18 months (mean, 9.6 months). The median number of single VPCs in repeat Holter was 3.5 per recording (range, 0-23 VPCs; no couplets or triplets) (Table 1).

In the unaffected group, VPCs were not detected in 10 of 32 dogs and only an infrequent number of single VPCs (median 2; range, 1-18 VPCs) was observed in the remaining 22 dogs. One dog had short period of supraventricular tachycardia, but the control Holter 6 months later did not show any abnormalities.

5 min six-lead electrocardiogram was also performed on all 46 dogs. During the 5-min electrocardiogram, three of the VA-affected group dogs showed $\geq 1$ VPC (range, 1-7 VPC) and one dog also 
M. Wiberg et al.

had ventricular couplets and triplets. One unaffected dog had a single VPC in 5-min electrocardiogram, but only one VPC was detected in Holter.

Table 2 shows the results of the PQ and QT interval measurements from the Holter recordings in the VA-affected, VA-borderline, and VAunaffected groups. There was no significant difference in QT intervals between the study groups.

\section{Echocardiography}

\section{Echocardiography was performed all 46 dogs}

Descriptive statistics of the echocardiography measurements of the 31 dogs in the VA-unaffected group are shown in Table 3. One unaffected dog was not included in the descriptive analysis because of mild MR (MR jet area/left atrium area $20 \%$ ). Echocardiography did not reveal any evidence of congenital or acquired heart disease in the other 31 unaffected dogs. Some of the echocardiographic variables were associated with gender (LVEDV, LVESV, LVIDd, EPSS, IVSd, left ventricular posterior wall thickness at end-systole, aorta diameter RPS SA view, and aorta hinge) and some variables with BW (LVEDV, LVESV, LVIDd, EPSS, IVSd, IVSs, sphericity index, and aorta hinge) and age (IVSs and sphericity index) $(p<0.05)$.

Based on multivariate analyses, for IVSd and EPSS, the gender effects remain significant or not significant ( $p$-values, 0.013 and 0.054 , respectively). For LVEDV, both gender and BW are not significant in the multivariate analysis ( $p$-values, 0.061 and 0.064 ). For LVESV and LVIDd, the $p$-values of both gender and BW increase to $>0.1$ in the multivariate analysis. In Table 3 the LVEDV, LVESV, LVIDd, and left ventricular internal dimension at end-systole results are described also separately for males and females.

Comparison of echocardiography variables between the VA-unaffected and VA-affected and
VA-borderline groups did not reveal any significant differences. Dog number 3 had a mild MR (MR jet area/left atrium area $20 \%$ ).

\section{Pedigree analysis}

The entire study population consisted of 39 litters with a total of 65 dogs that underwent clinical examination or necropsy. Of these litters, 23 included SCD or VA-affected dogs; 17 litters had SCD only, four litters had VA-affected individuals only, and two litters included both SCD and VA-affected dogs. In two of these litters, there were two and three SCD cases, respectively. Two littermates of SCD dogs were VA-affected.

Two composite pedigrees of 29 closely related litters (including 15 SCD dogs and 38 dogs from the clinical study, two of which died during follow-up) are illustrated in Fig. 2. Other, distantly related litters were not included. Unfortunately, full litters were not available for examination and the genealogical distribution of the data is thus biased. Consequently, the frequency of SCD and VA in the entire breed cannot be accurately estimated. Furthermore, multigenerational phenotypes were not available. On the basis of the available data, a definitive model of inheritance could not be determined.

\section{Discussion}

Our study describes VA as a cause of SCD in Leonbergers with $<3$ years of age. Necropsy showed no macroscopic or microscopic pathological changes in the heart. The postmortem findings were typical for acute cardiac arrest and arrhythmia was considered as the most likely cause for sudden death. To confirm the hypothesis of arrhythmia-related death, clinical evaluation with Holter monitoring was performed on young Leonbergers with and without a close family history of SCD. Holter

Table 2 Descriptive statistics of Holter ECG variables in Leonbergers by study group.

\begin{tabular}{lcccc}
\hline Variable & $\begin{array}{c}\text { VA-affected }(\mathrm{n}=7) \\
\text { mean }( \pm S D)\end{array}$ & $\begin{array}{c}\text { VA-borderline }(\mathrm{n}=7) \\
\text { mean }( \pm S D)\end{array}$ & $\begin{array}{c}\text { VA-unaffected }(\mathrm{n}=32) \\
\text { mean }( \pm S D)\end{array}$ & $p$ value \\
\hline Heart rate $(\mathrm{bpm})$ & $99.8( \pm 2.55)$ & $100.8( \pm 1.49)$ & $99.2( \pm 2.45)$ & 0.2280 \\
RR interval $(\mathrm{ms})$ & $600.3( \pm 14.37)$ & $592.9( \pm 10.48)$ & $603.3( \pm 15.35)$ & 0.2226 \\
PQ interval $(\mathrm{ms})$ & $116.6( \pm 17.58)$ & $115.0( \pm 18.80)$ & $110.9( \pm 9.68)$ & 0.5131 \\
QT interval $(\mathrm{ms})$ & $239.2( \pm 4.97)$ & $236.8( \pm 15.39)$ & $235.0( \pm 11.28)$ & 0.6257 \\
\hline
\end{tabular}

Mean 3 consecutive complexes were measured during Holter recording in heart rates 90 to 110 beats per minute (bpm). No significant difference was detected between study groups with any variable.

SD, standard deviation; ECG, electrocardiogram; VA, ventricular arrhythmia. 
Table 3 Descriptive statistics of echocardiographic measurements of 31 dogs unaffected by ventricular arrhythmias.

\begin{tabular}{|c|c|c|c|c|}
\hline Variable & Number & Mean & $\pm \mathrm{SD}$ & Range \\
\hline Age (months) ${ }^{a}$ & 31 & 20 & & $9-40$ \\
\hline $\mathrm{BW}(\mathrm{kg})^{\mathrm{a}}$ & 31 & 53.3 & & $40.9-72.0$ \\
\hline LVEDV (ml) & 31 & 94.9 & 15.04 & $68.0-126.0$ \\
\hline LVEDV male & 12 & 107.0 & 13.80 & $79.0-126.0$ \\
\hline LVEDV female & 19 & 87.2 & 10.07 & $68.0-111.0$ \\
\hline LVESV (ml) & 31 & 46.3 & 8.81 & $30.0-65.0$ \\
\hline LVESV male & 12 & 52.2 & 8.54 & $35.0-65.0$ \\
\hline LVESV female & 19 & 42.5 & 6.78 & $30.0-57.0$ \\
\hline LVEDVI $\left(\mathrm{ml} / \mathrm{m}^{2}\right)$ & 31 & 66.8 & 7.90 & $47.2-81.0$ \\
\hline LVESVI $\left(\mathrm{ml} / \mathrm{m}^{2}\right)$ & 31 & 32.6 & 5.29 & $22.6-45.2$ \\
\hline $\mathrm{EF}(\%)$ & 31 & 51.1 & 4.87 & $41.9-61.4$ \\
\hline LVIDd (mm) & 31 & 46.7 & 3.73 & $39.0-53.8$ \\
\hline LVIDd male & 12 & 49.3 & 3.39 & $43.0-53.8$ \\
\hline LVIDd female & 19 & 45.0 & 2.97 & $39.0-53.6$ \\
\hline LVIDs (mm) & 31 & 32.2 & 3.42 & $27.0-39.3$ \\
\hline LVIDs male & 12 & 33.4 & 3.62 & $27.0-39.3$ \\
\hline LVIDs female & 19 & 31.4 & 3.15 & $27.0-39.0$ \\
\hline IVSd (mm) & 31 & 11.9 & 0.87 & $10.5-13.9$ \\
\hline IVSs (mm) & 31 & 15.9 & 1.81 & $12.7-19.2$ \\
\hline LVPWd (mm) & 31 & 11.0 & 0.95 & $9.0-13.2$ \\
\hline LVPWs (mm) & 31 & 15.2 & 1.36 & $12.5-18.4$ \\
\hline $\mathrm{FS} \%$ & 31 & 31.2 & 4.61 & $24.3-40.9$ \\
\hline EPSS $(\mathrm{mm})^{\mathrm{a}}$ & 28 & 6.5 & & $5.0-8.5$ \\
\hline $\mathrm{Sl}^{\mathrm{a}}$ & 31 & 1.7 & & $1.4-2.2$ \\
\hline LA (mm) & 31 & 30.8 & 3.21 & $26.0-38.0$ \\
\hline Ao $(\mathrm{mm})$ & 31 & 26.6 & 2.69 & $21.0-32.0$ \\
\hline LA/Ao & 31 & 1.2 & 0.09 & $1.0-1.3$ \\
\hline Avel $(\mathrm{m} / \mathrm{s})^{\mathrm{a}}$ & 31 & 1.5 & & $1.2-2.3$ \\
\hline Pvel $(\mathrm{m} / \mathrm{s})^{a}$ & 31 & 1.2 & & $0.9-1.6$ \\
\hline Ao hinge $(\mathrm{mm})^{\mathrm{a}}$ & 29 & 23 & & $21.0-24.0$ \\
\hline
\end{tabular}

BW, body weight; LVEDV, left ventricular end-diastolic volume; LVESV, left ventricular end-systolic volume; LVEDVI, end-diastolic volume index; LVESVI, end-systolic volume index; EF, Simpson's ejection fraction; LVIDd, left ventricular internal diameter diastole; LVIDs, left ventricular internal diameter systole; IVSd, interventricular septum diastole; IVSs, interventricular septum systole; LVPWd, left ventricular posterior wall diastole; LVPWs, left ventricular posterior wall systole; FS, fractional shortening; EPSS, Epoint-to-septal separation; SI, sphericity index; LA, left atrial diameter; Ao, aortic root diameter; Avel, aortic peak velocity; Pvel, pulmonic peak velocity; RPS, right parasternal; SD, standard deviation.

${ }^{\text {a }}$ Variables not normally distributed and are presented as median and range.

monitoring demonstrated VA characterized by polymorphology and frequent complexity in a group of the apparently healthy dogs. Pedigree evaluation was suggestive of a familial disease. The term familial idiopathic VA can be used to describe this arrhythmia disorder in Leonbergers.

The larger number (20) of SCD cases identified in this study implies a significant health problem in
Leonbergers in Finland. Some litters had more than one dead. The typical age for SCD appears to be between 10 and 24 months; 14 of 20 dogs succumbed at this age. There was no difference between sexes. The dogs died suddenly without any preceding clinical signs, usually when they were resting or were at rest after exercise. When sudden death occurs without any cardiac or other changes, arrhythmia-related death can be suspected $[1,2]$. Ventricular arrhythmias are the most common arrhythmias that cause SCD [2,4,26,27]. Primary arrhythmia disorders in humans are usually familial and clinical and genetic screening of close relatives is important for determining cause of death and identifying individuals at risk $[1,2,26]$. In dogs, an early diagnosis of the familial disorder would also prevent use of affected dogs in breeding.

We observed that apparently healthy Leonbergers may be affected by malignant arrhythmia and that the arrhythmia disorder has a familial background. Typical for the severe form of arrhythmia was the presence of numerous fast ventricular couplets, triplets, and non-sustained periods of VT with polymorphic features. The number of single VPCs was usually only mildly to moderately increased. A tendency for ventricular complexity and polymorphology are considered to lead to increased risk for SCD, especially if there is a family history of unexplained death [26-29]. Two Leonbergers included in our clinical study died within 1 year after diagnosis of polymorphic VT. One of these two dogs had two full siblings with SCD, and the other dog had a littermate that was diagnosed with VT.

Our findings in Leonbergers resemble IVA reported in GSDs; young age of onset, sudden death at rest, and VA with features of polymorphic VTs without evidence of structural cardiac disease are typical [14,30]. Characteristically, the arrhythmias with Leonbergers were not related to the exercise or increased heart rates. In particular, ventricular complexity and VTs were found more frequently within slower heart rates and during sinus arrhythmia when the dogs were resting. Most of the VTs also preceded a longer RR interval and sinus pause; similar findings have been described with IVA in GSDs $[14,30]$.

The diagnosis of familial VA was based on Holter findings. Clinical signs were not observed by the owners even when the dog had severe VA. Short arrhythmia events are likely difficult to notice, especially if they occurred during rest. The value of 5-min electrocardiogram for screening VA was limited as arrhythmia occurred more as intermittent complexity than as an increased number of 


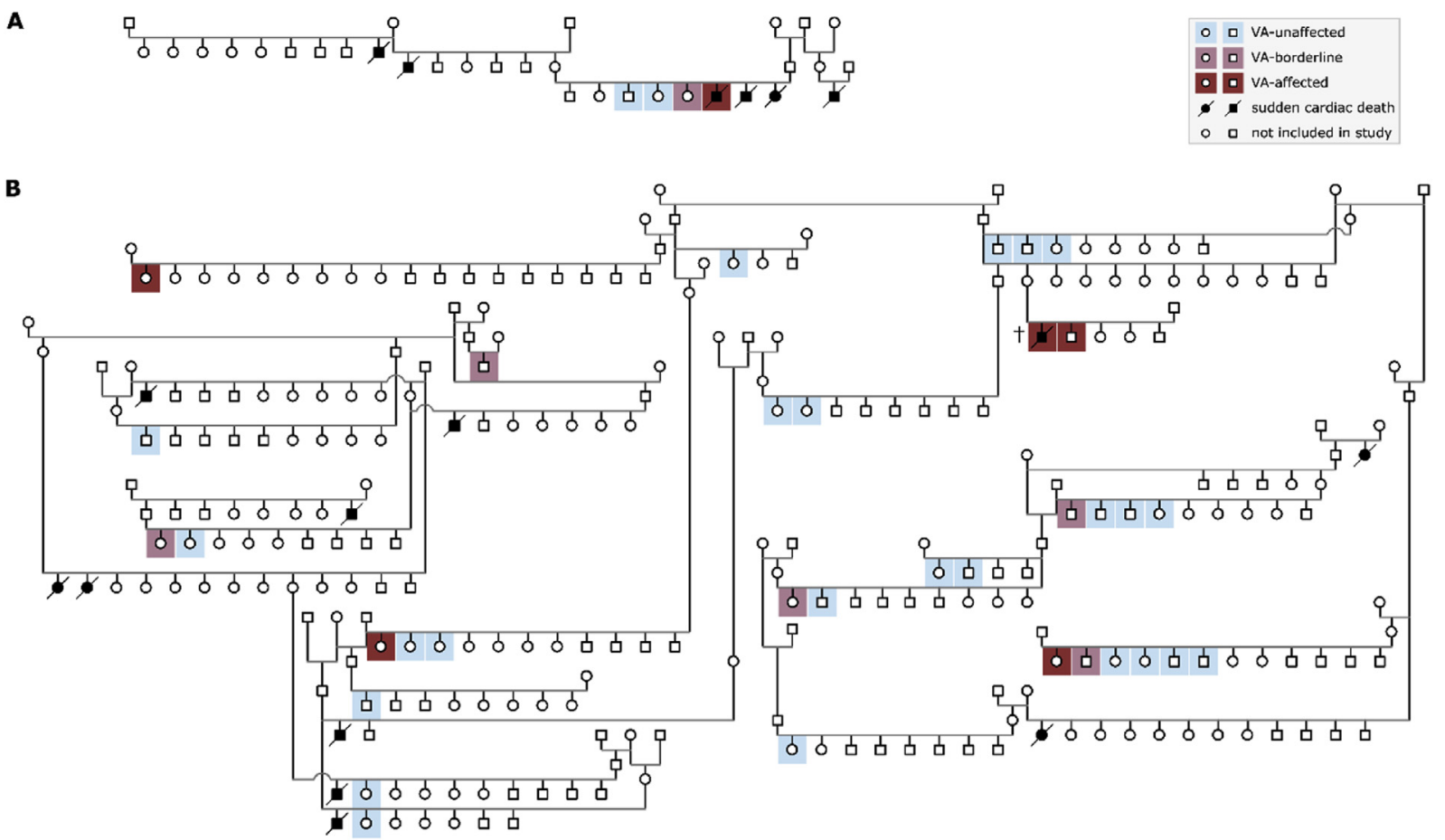

Fig. 2 Two composite pedigrees of the Leonbergers with sudden cardiac death (SCD) and dogs included in the present clinical study. Ventricular arrhythmia (VA) was diagnosed with Holter monitoring. Dogs were classified as VAaffected (dark red symbol), VA-borderline affected (pale red), and VA-unaffected (light blue) in accordance with VA severity. Dogs with SCD are marked with a black symbol. The VA-affected dog marked with a dagger ( $\dagger$ ) was euthanized.

single VPCs. Echocardiography did not reveal any significant findings but should nevertheless be part of the clinical evaluation of suspected arrhythmia cases to exclude possible congenital anomalies or cardiomyopathies.

Serum troponin $\mathrm{I}$ is a marker for myocardial damage and is elevated in DCM in Dobermann and arrhythmogenic cardiomyopathy in Boxer with VAs even in the absence of echocardiographic changes [12,31-33]. Troponin I has also shown to be useful in SCD risk assessment with DCM in Doberman [11]. We did not find that high-sensitivity $\mathrm{Tnl}$ is diagnostic for VA or correlated with the severity of arrhythmia in Leonbergers. This further supports our current evidence that Leonbergers have a primary arrhythmia rather than myocardial disease. Increased hs-Tnl was found only in one severely affected dog. This dog was clinically healthy until euthanasia 8 months after VA diagnosis. Unfortunately, we were not able to follow the hs-Tnl levels in this dog. A longer follow-up period with repeated measurements would have been necessary to evaluate the usefulness of hsTnl for risk assessment of SCD.

The VA in Leonbergers may be challenging to diagnose because of variations in phenotype severity and age-related occurrence. Although arrhythmia characteristics include the presence of ventricular complexity, Holter findings varied from a single couplet to frequent periods of VTs. Previous studies have shown that fast ventricular couplets, triplets, and non-sustained VTs are uncommon with healthy dogs and should be considered a sign of an increased risk for cardiovascular events [7,34-36]. In this study, we classified dogs as borderline affected if only a single fast couplet or triplet was detected. No VA complexity was detected in the control Holter monitoring of these borderline cases. Ventricular arrhythmias may have a remarkable day-to-day variation and it is possible that infrequent complexes are not present every day [36]. It is also possible that a small number of couplets or triplets are a normal finding for this breed. Thus, misclassification may occur especially with the milder VA phenotypes and the diagnostic criteria for Leonbergers requires further evaluation.

In humans, polymorphic VTs without evidence of a structural cardiac disease are commonly associated with cardiac channelopathies [1,4,26,28]. Primary channelopathies are usually familial and 
characterized by different phenotypes and incomplete penetrance $[3,4]$. The most common channelopathy is long QT syndrome, a disorder of delayed ventricular repolarization that may predispose to a life-threatening VT called Torsades de pointes, which is characterized by waxing and waning QRS amplitude $[3,4,26,28]$. In dogs, reports of primary QT interval abnormalities are sparse and the diagnostic criteria have not been validated $[14,16,37]$. In humans, QT intervals are calculated by using specific correction formulas, which reduce the effect of $H R[1,4,37]$. In our study, to avoid the effect of HR on QT intervals, we calculated the mean QT within the same HR range (90-110 bpm) using the Holter recordings [14]. We did not identify any differences between the QT intervals between the VA-affected and the VAunaffected group. Channelopathies are, however, an attractive explanation for VA in Leonbergers and further studies on the mechanism of VA are warranted. In humans, many VTs previously considered idiopathic have later been identified as channelopathies [4].

The breed specificity and high prevalence of VA and SCDs in Leonbergers indicate that there is a strong genetic background to the disease. However, based on the available pedigree data, a definitive mode of inheritance could not be determined. In particular, without knowledge of the phenotypes of the parents of the affected litters, it can only be speculated whether the underlying genetic defect is inherited in a recessive or dominant manner or if disease penetrance is incomplete. Assessing the inheritance model of this disease is challenging because of the early age of onset. Ideally, parents and litters should be clinically examined with Holter monitoring over multiple generations and before the age of 3 years to ensure that potential arrhythmias are detected appropriately. Although this could not be achieved within the scope of our study, it is nevertheless of critical importance in defining the mode of inheritance. Currently, genetic analyses to uncover the disease-causing variants are ongoing and may reveal the inheritance mechanism and elucidate the molecular and pathophysiological pathways.

A limitation of present study is the short followup period of the clinical study. It is unclear whether the severity of arrhythmias will decrease if the dogs included to this study survive to an older age. With
IVA in GSD, it has been shown that spontaneous resolution of the VA may occur with age [30]. A previous study ${ }^{k}$ reported arrhythmias in a group of older Leonbergers (mean age, 6.0 years), but at least some of these dogs had also evidence of structural cardiac diseases. Thus, it is more likely that the arrhythmias detected in the older Leonbergers are of different etiology. Using 24-h Holter instead of 48 -h or 7 days monitoring can also be considered as a limitation of this study. However, if multiday recording is not available, repeated 24-h Holter monitoring is recommended to diagnose and follow-up VA in Leonbergers. Based on our findings, VAs characterized by frequent complexity is a significant risk factor for sudden death in young Leonbergers. Other currently unidentified prognostic factors may exist. Further follow-up of the remaining dogs included in this study by Holter monitoring and echocardiography may provide more exact information of the prognosis.

\section{Conclusion}

We have described malignant VA associated with SCD in young Leonbergers. Pedigree evaluation was suggestive of a familial disease, and screening for VA is thus recommended for close relatives of SCD dogs. The arrhythmia appears to be age-related; Holter monitoring is recommended before 3 years of age.

\section{Conflicts of Interest Statement}

The authors do not have any conflicts of interest to disclose.

\section{Acknowledgments}

The authors would like to thank Laura Parikka for technical assistance and Jouni Niemi, Vetman Oy, Finland for technical support with the Televet Holter device, and biostatistician Jouni Junnila for his contribution to statistical analyses. We would also like to thank all the owners of the Leonbergers for bringing their dogs to participate in the study. This study was funded by Doctoral Programme in Integrative Life Science (ILS), University of Helsinki; Jane and Aatos Erkko Foundation, Helsinki;

\footnotetext{
${ }^{k}$ Ribas T, Bublot $\mathrm{P}$, Thireau B, Rannou A, Thomas A, Cadore JL, Pariaut R. Arrhythmias in a cohort of Leonbergers. British Small Animal Veterinary Congress, April 3-6, 2014, Birmingham, UK. Abstract.
} 
Q4 Academy of Finland, Helsinki and HiLIFE, University of Helsinki, Finland.

\section{References}

[1] Priori SG, Blomström-Lundqvist C, Mazzanti A, Blom N, Borggrefe M, Camm J, Elliott PM, Fitzsimons D, Hatala R, Hindricks G, Kirchhof P, Kjeldsen K, Kuck K-H, Hernandez A, Nikolaou N, Norekval TM, Spaulding C, Van Veldhuisen DJ. ESC Guidelines for the management of patients with ventricular arrhythmias and the prevention of sudden cardiac death. The task force for the management of patients with ventricular arrhythmias and the prevention of sudden cardiac death of the European Society of Cardiology (ESC).Endorsed by: association for European Paediatric and Congenital Cardiology (AEPC). Eur Heart J 2015;36:2793-867. 2015.

[2] Delaney RJ, Gallagher PJ. The pathological investigation of sudden cardiac death. Diagn Histopathol 2017;23: 449-509.

[3] Fernández-Falgueras A, Sarquella-Brugada G, Brugada J, Brugada R, Campuzano O. Review: cardiac channelopathies and sudden death: recent clinical and genetic advances. Biology 2017;6:7.

[4] Garcia-Elias A, Benito B. Ion channel disorders and sudden cardiac death. Int J Mol Sci 2018;19:692.

[5] Kienle RD, Thomas WP, Pion PD. The natural clinical history of canine congenital subaortic stenosis. J Vet Intern Med 1994;8:423-31.

[6] Calvert CA, Hall G, Jacobs G, Pickus C. Clinical and pathologic findings in Doberman Pinschers with occult cardiomyopathy that died suddenly or developed congestive heart failure: 54 cases (1984-1991). J Am Vet Med Assoc 1997;210:505-11.

[7] Basso C, Fox PR, Meurs KM, Towbin JA, Spier AW, Calabrese F, Maron BJ, Thiene G. Arrhythmogenic right ventricular cardiomyopathy causing sudden cardiac death in Boxer dogs. A new animal model of human disease. Circulation 2004;109:1180-5.

[8] Martin MWS, Stafford Johnson MJ, Celona B. Canine dilated cardiomyopathy: a retrospective study of signalment, presentation and clinical findings in 369 cases. J Small Anim Pract 2009;50:23-9.

[9] Wess G, Schulze A, Butz V, Simak J, Killich M, Keller LJM, Maeurer J, Hartmann K. Prevalence of dilated cardiomyopathy in Doberman Pinschers in various age groups. J Vet Intern Med 2010;24:533-8.

[10] Meurs KM. Genetics of cardiac disease in the small animal patient. Vet Clin North Am Small Anim Pract 2010;40: 701-15.

[11] Kluser L, Holler PJ, Simak J, Tater G, Smets P, Rugamer D, Kuchenhoff $H$, Wess $G$. Predictors of sudden cardiac death in Doberman Pinschers with dilated cardiomyopathy. J Vet Intern Med 2016;30:722-32.

[12] Meurs K. Arrhythmogenic right ventricular cardiomyopathy in the Boxer dog: an update. Vet Clin North Am Small Anim Pract 2017;47:1103-11.

[13] Dambach DM, Lannon A, Sleeper MM, Buchanan J. Familial dilated cardiomyopathy of young Portuguese Water Dogs. J Vet Intern Med 1999;13:65-71.

[14] Moise NS, Meyers-Wallen V, Flahive WJ, Valentine A, Scarlett JM, Brown CA, Chavkin MJ, Dugger DA, RenaudFarrell S, Kornreich B, Schoenbron WC, Sparks JR, Gilmour RF. Inherited ventricular arrhythmias and sudden death in German Shepherd dogs. J Am Coll Cardiol 1994; 24:233-43.

[15] Meurs KM, Weidman JA, Rosenthal SL, Lahmers KK, Friedenberg SG. Ventricular arrhythmias in Rhodesian Ridgebacks with a family history of sudden death and results of a pedigree analysis for potential inheritance patterns. J Am Vet Med Assoc 2016;15:1135-8.

[16] Ware WA, Reina-Doreste Y, Stern JA, Meurs KM. Sudden death associated with QT interval prolongation and KCNQ1 gene mutation in a family of English Springer Spaniels. J Vet Intern Med 2015;29:561-8.

[17] Sahn DJ, DeMaria A, Kisslo J, Weyman A. Recommendations regarding quantitation in M-mode echocardiography: results of a survey of echocardiographic measurements. Circulation 1978;58:1072-83.

[18] Thomas WP, Gaber CE, Jacobs GJ, Kaplan PM, Lombard CW, Moise NS, Moses BL. Recommendations for standards in transthoracic two-dimensional echocardiography in the dog and cat. Echocardiography committee of the specialty of cardiology, American college of veterinary internal medicine. J Vet Intern Med 1993;7:247-52.

[19] Lang RM, Badano LP, Mor-Avi V, Afilalo J, Armstrong A, Ernande L, Flachskampf FA, Foster E, Goldstein SA, Kuznetsova T, Lancellotti P, Muraru D, Picard MH, Rietzschel ER, Rudski L, Spencer KT, Tsang W, Voigt J-U. Recommendations for chamber quantification by echocardiography in adults: an update from the American society of echocardiography and European association of cardiovascular imaging. Eur Heart J - Cardiovasc Imaging 2015;16:233-71.

[20] Wess G, Maeurer J, Simak J, Hartmann K. Use of Simpson's method of disc to detect early echocardiographic changes in Doberman Pinschers with dilated cardiomyopathy. J Vet Intern Med 2010;24:1069-76.

[21] Hansson K, Häggström J, Kvart C, Lord P. Left atrial to aortic root indices using two-dimensional and M-mode echocardiography in Cavalier King Charles Spaniels with and without left atrial enlargement. Vet Radiol Ultrasound 2002;43:568-75.

[22] Abbott JA, MacLean HN. Comparison of Doppler-derived peak aortic velocities obtained from subcostal and apical transducer sites in healthy dogs. Vet Radiol Ultrasound 2003;44:695-8.

[23] Muzzi RA, de Araújo RB, Muzzi LAL, Pena JLB, Silva EF. Regurgitant jet area by Doppler color flow mapping: quantitative assessment of mitral regurgitation severity in dogs. J Vet Cardiol 2003;5:33-8.

[24] Niskanen J, Salmela E, Lohi H. AncesTrim - a tool for trimming complex pedigrees. J Open Source Softw 2017;2: 179. https://doi.org/10.21105/joss.00179.

[25] Turk JR, Turk MAM, Root CR. Necropsy of the canine heart: a simple technique for quantifying ventricular hypertrophy and valvular alterations. Comp Cont Ed Pract Vet 1983;5: 905-10.

[26] Al-Khatib SM, Stevenson WG, Ackerman MJ, Bryant WJ, Callans DJ, Curtis AB, Deal BJ, Dickfeld T, Field ME, Fonarow GC, Gillis AM, Granger CB, Hammill SC, Hlatky MA, Joglar JA, Kay GN, Matlock DD, Myerburg RJ, Page RL. AHA/ACC/HRS guideline for management of patients with ventricular arrhythmias and the prevention of sudden cardiac death: a report of the American College of Cardiology/American Heart Association task force on clinical practice guidelines and the Heart Rhythm Society. J Am Coll Cardiol 2017;72:91-220. 2017.

[27] Nagiub M, Carter K, Shepard R. Systematic review of risk stratification of pediatric ventricular arrhythmia in 
structurally normal and abnormal hearts. Prog Pediatr Cardiol 2017;45:55-62.

[28] Prystowsky EN, Padanilam BJ, Joshi S, Fogel RI. Ventricular arrhythmias in the absence of a structural heart disease. J Am Coll Cardiol 2012;59:1733-44.

[29] Ephrem G, Levine M, Friedmann P, Schweitzer P. The prognostic significance of frequency and morphology of premature ventricular complexes during ambulatory Holter monitoring. Ann Noninvasive Electrocardiol 2013;18: 118-25.

[30] Moise NS, Gilmour RF, Riccio ML. An animal model of spontaneous arrhythmic death. J Cardiovasc Electrophysiol 1997;8:98-103.

[31] Baumwart RD, Orvalho J, Meurs KM. Evaluation of serum cardiac troponin I concentration in Boxers with arrhythmogenic right ventricular cardiomyopathy. Am J Vet Res 2007;68:524-8.

[32] Wess G, Simak J, Mahling M, Hartmann K. Cardiac troponin I in Doberman Pinschers with cardiomyopathy. J Vet Intern Med 2010;24:843-9.
[33] Kluser L, Maier ET, Wess G. Evaluation of a high-sensitivity cardiac troponin I assay compared to a first-generation cardiac troponin I assay in Doberman Pinschers with and without dilated cardiomyopathy. J Vet Intern Med 2018;33: 54-63.

[34] Calvert CA, Jacobs GJ, Smith DD, Rathbun SL, Pickus C. Association between results of ambulatory electrocardiography and development of cardiomyopathy during long-term follow-up of Doberman Pinschers. J Am Vet Med Assoc 2000;216:34-9.

[35] Calvert CA, Jacobs G, Pickus CW, Smith DD. Results of ambulatory electrocardiography in overtly healthy Doberman Pinschers with echocardiographic abnormalities. J Am Vet Med Assoc 2000;217:1328-32.

[36] Meurs KM, Spier AW, Wright NA, Hamlin RL. Use of ambulatory electrocardiography for detection of ventricular premature complexes in healthy dogs. J Am Vet Med Assoc 2001;218:1291-2.

[37] Fossa AA. Assessing QT prolongation in conscious dogs: validation of a beat-to-beat method. Pharmacol Ther 2008;18:231-8.

Available online at www.sciencedirect.com

\section{ScienceDirect}

